\title{
CONVERGENCE OF CERTAIN COSINE SUMS IN THE METRIC SPACE $L$
}

\author{
BABU RAM
}

\begin{abstract}
We consider here the $L^{1}$ convergence of Rees-Stanojevic cosine sums to a cosine trigonometric series belonging to the class $S$ defined by Sidon and deduce as corollaries some previously known results from our result.
\end{abstract}

1. Introduction. Sidon [6] introduced the following class of cosine trigonometric series: Let

$$
\frac{a_{0}}{2}+\sum_{k=1}^{\infty} a_{k} \cos k x
$$

be a cosine series satisfying $a_{k}=o(1), k \rightarrow \infty$. If there exists a sequence $\left\{A_{k}\right\}$ such that

$$
\begin{gathered}
A_{k} \downarrow 0, \quad k \rightarrow \infty, \\
\sum_{k=0}^{\infty} A_{k}<\infty, \\
\left|\Delta a_{k}\right| \leqslant A_{k}, \quad \forall k,
\end{gathered}
$$

we say that (1.1) belongs to the class $S$.

Let the partial sums of (1.1) be denoted by $S_{n}(x)$ and $f(x)=\lim _{n \rightarrow \infty} S_{n}(x)$.

Recently, Garrett and Stanojević [3] proved that the partial Rees-Stanojević sums [5]

$$
g_{n}(x)=\frac{1}{2} \sum_{k=0}^{n} \Delta a_{k}+\sum_{k=1}^{n} \sum_{j=k}^{n} \Delta a_{j} \cos k x
$$

converge in the $L^{1}$ metric to (1.1) if and only if given $\varepsilon>0$, there is a $\delta(\varepsilon)>0$ such that

$$
\int_{0}^{\delta}\left|\sum_{k=n+1}^{\infty} \Delta a_{k} D_{k}(x)\right| d x<\varepsilon
$$

for all $n \geqslant 0$. It has been shown in the same paper that the classical Young-Kolmogorov-Stanojević sufficient conditions for integrability of (1.1) imply (1.6).

Received by the editors October 7, 1976.

AMS (MOS) subject classifications (1970). Primary 42A20, 42 A32.

Key words and phrases. $L^{1}$ convergence of cosine sums, the class $S$, quasi-convex sequence.

○ American Mathematical Society 1977 
Generalising a classical result [1, p. 204], Teljakovskii [7] proved the following.

THEOREM A. If (1.1) belongs to the class $S$, then a necessary and sufficient condition for $L^{1}$ convergence of (1.1) is $a_{n} \log n=o(1), n \rightarrow \infty$.

2. Lemmas. The proofs of our results are based upon the following lemmas.

LEMMA 1 (Fomin [2]). If $\left|c_{k}\right| \leqslant 1$, then

$$
\int_{0}^{\pi}\left|\sum_{k=0}^{n} c_{k} \frac{\sin (k+1 / 2) x}{2 \sin x / 2}\right| d x<C(n+1),
$$

where $C$ is a positive absolute constant.

LEMMA 2. If (1.1) belongs to the class $S$, then

$$
g_{n}(x)=S_{n}(x)-a_{n+1} D_{n}(x),
$$

where $D_{n}(x)$ denotes the Dirichlet kernel.

Proof. Since (1.1) belongs to the class $S$, we have

$$
a_{k} \rightarrow 0 \text { and } \sum_{k=0}^{\infty}\left|\Delta a_{k}\right|<\infty .
$$

The conditions of Lemma 1 of Garrett and Stanojevic [3] are thus satisfied and the result follows.

3. Main result. The main result of this paper reads:

THEOREM. If (1.1) belongs to the class $S$, then (1.6) holds. Hence

$$
\left\|f-g_{n}\right\|_{L^{1}}=o(1), \quad n \rightarrow \infty .
$$

Proof. Making use of Abel's transformation and Lemma 1, we have

$$
\begin{aligned}
\int_{0}^{\pi}\left|f(x)-g_{n}(x)\right| d x & =\int_{0}^{\pi}\left|\sum_{k=n+1}^{\infty} \Delta a_{k} D_{k}(x)\right| d x \\
& =\int_{0}^{\pi}\left|\sum_{k=n+1}^{\infty} A_{k} \frac{\Delta a_{k}}{A_{k}} D_{k}(x)\right| d x \\
& =\int_{0}^{\pi}\left|\sum_{k=n+1}^{\infty} \Delta A_{k} \sum_{i=0}^{k} \frac{\Delta a_{i}}{A_{i}} D_{i}(x)\right| d x \\
& \leqslant \sum_{k=n+1}^{\infty} \Delta A_{k} \int_{0}^{\pi}\left|\sum_{i=0}^{k} \frac{\Delta a_{i}}{A_{i}} D_{i}(x)\right| d x \\
& \leqslant C \sum_{k=n+1}^{\infty}(k+1) \Delta A_{k} .
\end{aligned}
$$

(1.2) and (1.3) now imply the conclusion of the Theorem. 
4. Corollaries. (i) Using Lemma 2, we notice that

$$
\begin{aligned}
\int_{0}^{\pi}\left|f(x)-S_{n}(x)\right| d x & =\int_{0}^{\pi}\left|f(x)-g_{n}(x)+g_{n}(x)-S_{n}(x)\right| d x \\
& \leqslant \int_{0}^{\pi}\left|f(x)-g_{n}(x)\right| d x+\int_{0}^{\pi}\left|g_{n}(x)-S_{n}(x)\right| d x \\
& \leqslant \int_{0}^{\pi}\left|f(x)-g_{n}(x)\right| d x+\int_{0}^{\pi}\left|a_{n+1} D_{n}(x)\right| d x
\end{aligned}
$$

and

$$
\begin{aligned}
& \int_{0}^{\pi}\left|a_{n+1} D_{n}(x)\right| d x=\int_{0}^{\pi}\left|g_{n}(x)-S_{n}(x)\right| d x \\
& \leqslant \int_{0}^{\pi}\left|f(x)-S_{n}(x)\right| d x+\int_{0}^{\pi}\left|f(x)-g_{n}(x)\right| d x .
\end{aligned}
$$

Since $\lim _{n \rightarrow \infty} \int_{0}^{\pi}\left|f(x)-g_{n}(x)\right| d x=0$ by our Theorem and $\int_{0}^{\pi}\left|a_{n+1} D_{n}(x)\right| d x$ behaves like $a_{n+1} \log n$ for large values of $n$, Theorem $\mathrm{A}$ of Teljakovskii follows.

(ii) Let $a_{k} \rightarrow 0$ and $\sum_{k=1}^{\infty}(k+1)\left|\Delta^{2} a_{k}\right|<\infty$. Then $g_{n}$ converges to $f$ in the metric space $L$ since the trigonometric cosine series (1.1) with quasi-convex coefficients belongs to the class $S$ if we choose $A_{k}=\sum_{m=k}^{\infty}\left|\Delta^{2} a_{m}\right|$. This is Example 1 of [3].

5. Remark. In [4], Garrett and Stanojević proved (Corollary B, p. 70) that their Theorem B extends the Teljakovskii result.

My thanks are due to the referee for his wise comments which have definitely improved the presentation of this paper.

\section{REFERENCES}

1. N. K. Bari, A treatise on trigonometric series, Vol. II, Pergamon Press, London; Macmillan, New York, 1964, p. 204.

2. G. A. Fomin, On linear methods for summing Fourier series, Mat. Sb. 66 (107) (1964), 114-152.

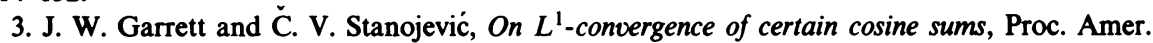
Math. Soc. 54 (1976), 101-105.

4. _ Necessary and sufficient conditions for $L^{1}$ convergence of trigonometric series, Proc. Amer. Math. Soc. 60 (1976), 68-71.

5. C. S. Rees and C. V. Stanojevic, Necessary and sufficient condition for integrability of certain cosine sums, J. Math. Anal. Appl. 43 (1973), 579-586.

6. S. Sidon, Hinreichende Bedingungen für den Fourier-Charakter einer trigonometrischen Reihe, J. London Math. Soc. 14 (1939), 158-160.

7. S. A. Teljakovskii, A certain sufficient condition of Sidon for the integrability of trigonometric series, Mat. Zametki 14 (1973), 317-328.

Department of Mathematics, Rohtak University, Rohtak-124001, INDia 